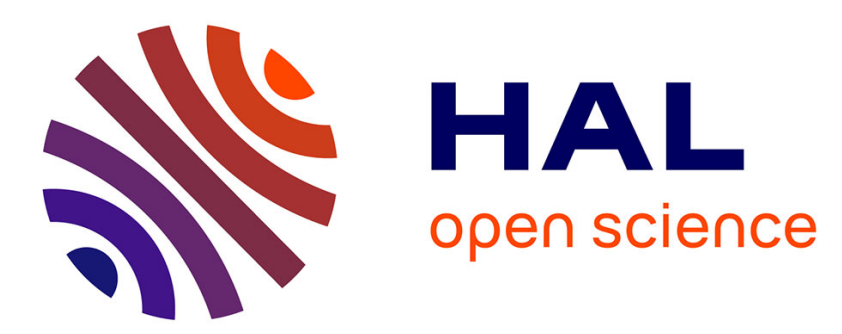

\title{
Spectre d'absorption dans l'infrarouge lointain en liaison avec leurs structures des composés Se1-xGe x amorphes
}

\author{
J.M. Laurant, A. Ribeyron
}

\section{To cite this version:}

J.M. Laurant, A. Ribeyron. Spectre d'absorption dans l'infrarouge lointain en liaison avec leurs structures des composés Se1-xGe x amorphes. Revue de Physique Appliquée, 1977, 12 (5), pp.759761. 10.1051/rphysap:01977001205075900 . jpa-00244237

\section{HAL Id: jpa-00244237 https://hal.science/jpa-00244237}

Submitted on 1 Jan 1977

HAL is a multi-disciplinary open access archive for the deposit and dissemination of scientific research documents, whether they are published or not. The documents may come from teaching and research institutions in France or abroad, or from public or private research centers.
L'archive ouverte pluridisciplinaire HAL, est destinée au dépôt et à la diffusion de documents scientifiques de niveau recherche, publiés ou non, émanant des établissements d'enseignement et de recherche français ou étrangers, des laboratoires publics ou privés. 


\section{SPECTRE D'ABSORPTION DANS L'INFRAROUGE LOINTAIN EN LIAISON AVEC LEURS STRUCTURES DES COMPOSÉS $\mathrm{Se}_{1-x} \mathrm{Ge}_{x}$ AMORPHES}

\section{J. M. LAURANT et A. RIBEYRON}

C. R. T. B. T., Centre de Recherches sur les Très Basses Températures, 166 X, 38042 Grenoble Cedex, France

Résumé. - L'absorption entre $10 \mathrm{~cm}^{-1}$ et $110 \mathrm{~cm}^{-1}$ des composés $\mathrm{Se}_{1-x} \mathrm{Ge}_{x}$ a été mesurée à $4,2 \mathrm{~K}$ pour $x=0 \%, 1 \%, 4 \%, 10 \%, 16 \%, 20 \%, 30 \%$. Un modèle est développé permettant de suivre l'évolution de la proportion d'anneaux et de chaînes de sélénium et de tétraèdres $\mathrm{GeSe}_{4}$ dans les alliages $\mathrm{Se}_{1-x} \mathrm{Ge}_{x}$.

Abstract. - The optical absorption of amorphous $\mathrm{Se}_{1-x} \mathrm{Ge}_{x}$ has been measured in the range 10 to $110 \mathrm{~cm}^{-1}$ at $4.2 \mathrm{~K}$. A model is developed to follow the evolution of proportion of selenium's chains, rings and tetrahedres $\mathrm{GeSe}_{4}$ into compounds $\mathrm{Se}_{1-x} \mathrm{Ge}_{x}(x$ from $0 \%$ to $30 \%$ ).

Dans les cristaux, la présence de symétries cristallines ne permet qu'à un petit nombre de modes au centre de la zone de Brillouin de contribuer au spectre d'absorption. Par contre dans les matériaux amorphes la disparition des symétries donne la possibilité du couplage des ondes électromagnétiques avec tous les modes de vibrations optiques et acoustiques [1].

La caractéristique des spectres pour $x=0 \%, 1 \%$ et $4 \%$, (Figs. 1,2) est l'existence de deux pics d'absorption, l'un vers $62 \mathrm{~cm}^{-1}$, l'autre vers $102 \mathrm{~cm}^{-1}$ [2]. L'intensité de ces pics décroît lorsque la concentration en Ge augmente.

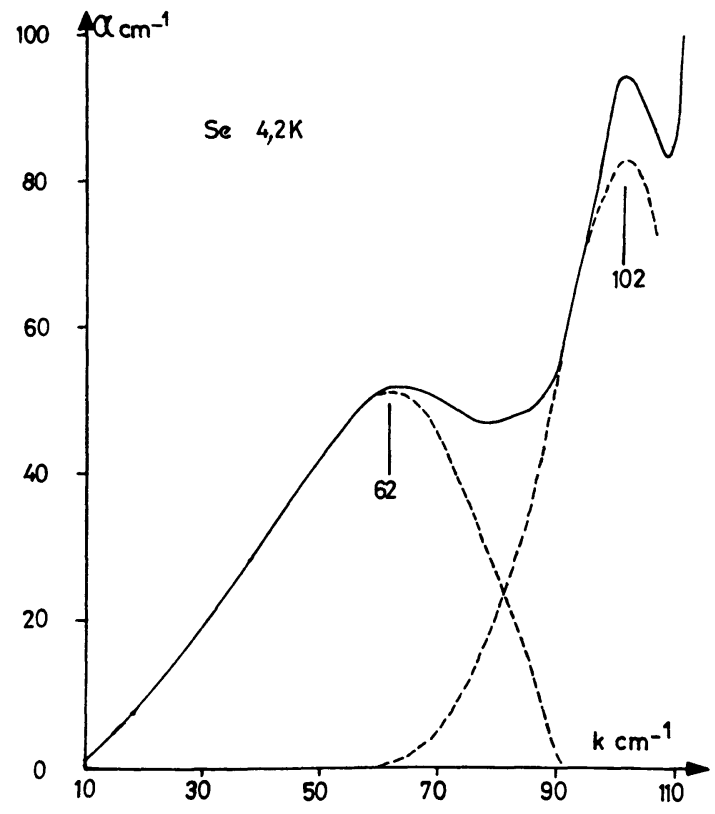

FIG. 1. - Décomposition du spectre d'absorption de Se amorphe.

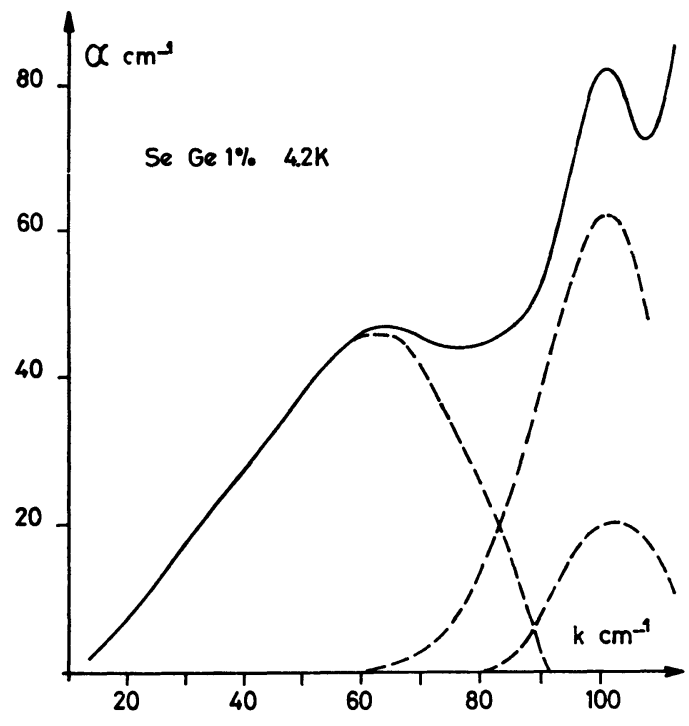

FIG. 2. - Décomposition du spectre d'absorption de $\mathrm{Se}_{0,99} \mathrm{Ge}_{0,01}$ amorphe.

La caractéristique des spectres pour $x=10 \%, 16 \%$ $20 \%, 30 \%$, (Figs. 3, 4) est l'existence d'un seul pic bien défini à $103 \mathrm{~cm}^{-1}$. L'intensité de ce pic croît de manière linéaire avec la concentration en Ge.

Le sélénium existe sous deux formes principales à l'état cristallin : trigonal (chaînes hélicoïdales) et monoclinique (anneaux $\mathrm{Se}_{8}$ ). Le pic à $102 \mathrm{~cm}^{-1}$ est le pic trouvé par Lucovsky [3] dans le sélénium monoclinique ; il a la même dépendance en fréquence suivant la température : $97 \mathrm{~cm}^{-1}$ à $300 \mathrm{~K}$ et $102 \mathrm{~cm}^{-1}$ à $4,2 \mathrm{~K}$. Le pic à $62 \mathrm{~cm}^{-1}$, nous l'interprétons comme étant le pic du mode de rotation des chaînes (dans le cristal trigonal un mode à $112 \mathrm{~cm}^{-1}$ a été attribué à cette 


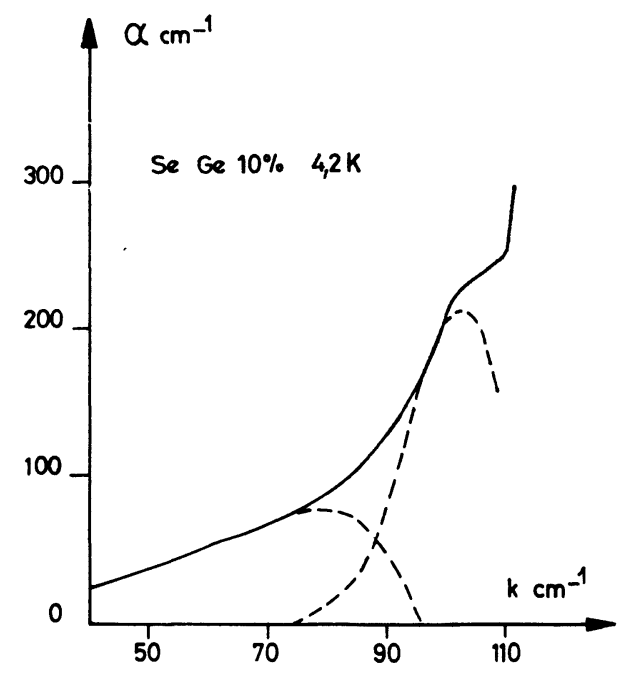

FIG. 3. - Décomposition du spectre d'absorption de $\mathrm{Se}_{0,9} \mathrm{Ge}_{0,1}$ amorphe.

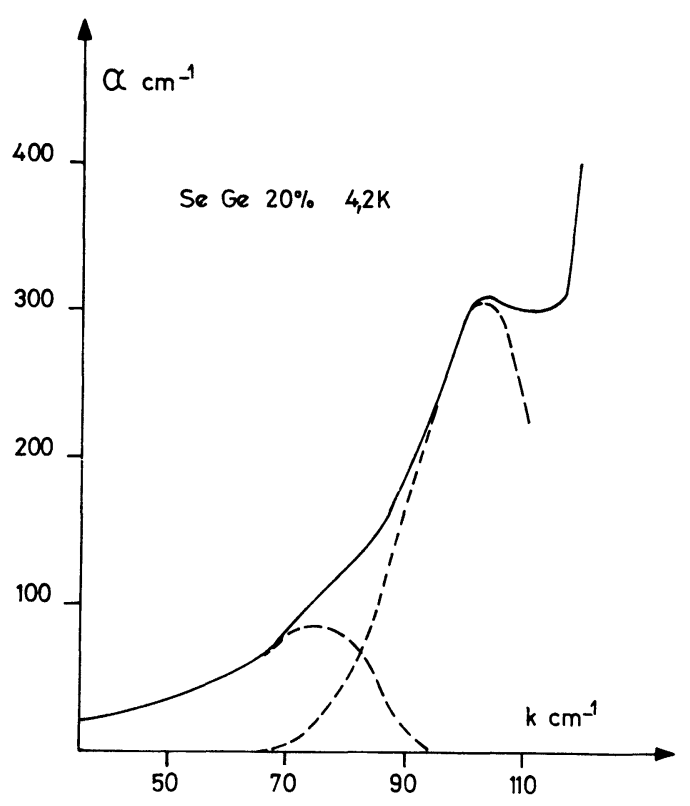

FIG. 4. - Décomposition du spectre d'absorption de $\mathrm{Se}_{0,8} \mathrm{Ge}_{0,2}$ amorphe.

vibration). Ce mode est très dépendant des liaisons interchaînes et indépendant des liaisons intrachaînes ; si l'on compare grâce aux mesures de diffraction des rayons $X$ [4] les distances interatomiques entre $1^{\text {er }}$ voisins et seconds voisins on a $2,37 \AA$ et $3,45 \AA$ dans le cristal trigonal, alors que dans le sélénium amorphe, on a respectivement $2,35 \AA$ et $3,75 \AA$. On constate que les liaisons interchaînes sont considérablement allongées dans le Se amorphe, donc l'énergie d'interaction entre chaînes va fortement diminuer. Un calcul montre, en admettant que l'on ait un potentiel d'interaction de Van der Vals entre chaînes, que le pic à $102 \mathrm{~cm}^{-1}$ peut décroître jusqu'à $60 \mathrm{~cm}^{-1}$.

Pour les alliages $\mathrm{Se}_{1-x} \mathrm{Ge}_{x}(x=1 \%$ et $4 \%$ ) les pics à 102 et $62 \mathrm{~cm}^{-1}$ diminuent, aucun nouveau pic bien défini n'apparaît dans notre zone de fréquence, donc le nombre de chaînes et d'anneaux de Se diminuent.

Pour les alliages $\mathrm{Se}_{1-x} \mathrm{Ge}_{x}(x=10 \%, 16 \%, 20 \%$, $30 \%$ ) le pic apparaissant à $103 \mathrm{~cm}^{-1}$ est attribué un mode de vibration moléculaire du tétraèdre $\mathrm{GeSe}_{4}$. La fréquence est calculée par rapport aux molécules du même type $\mathrm{GeCl}_{4}, \mathrm{GeI}_{4}, \mathrm{GeS}_{4}$.

Pour la concentration $x=10 \%$ un léger épaulement apparaît à $40 \mathrm{~cm}^{-1}$ dans la courbe d'absorption. Si l'on trace la courbe $\alpha / k^{2}$ en fonction de $k$, on peut retirer la composante acoustique (absorption proportionnelle à $k^{2}$ ). Celle-ci est inexistante dans le Se pur mais présente en général dans les verres à structures tétraédriques, tel $\mathrm{GeO}_{2}, \mathrm{SiO}_{2}[1,5]$. En traçant ce texcès en $\alpha(\mathrm{k})$, (Fig. 5) on trouve un pic bien défini à $40 \mathrm{~cm}^{-1}$. Ce pic

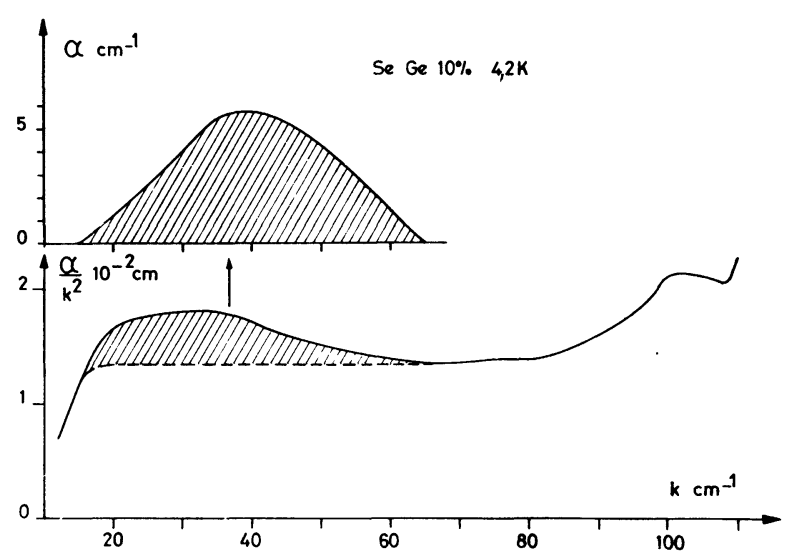

FIG. 5. - Détermination des modes en excès à basse fréquence pour $\mathrm{Se}_{0,9} \mathrm{Ge}_{0,1}$.

d'absorption a été attribué aux chaînes libres; en effet, d'après les mesures de diffraction de Rayons $X$ pour cette concentration, les distances aux seconds voisins augmentent jusqu'à $3,80 \AA$, ce qui explique le déplacement du pic attribué au mode de rotation des chaînes libres de $62 \mathrm{~cm}^{-1}$ à $40 \mathrm{~cm}^{-1}$.

Pour la concentration $x=16 \%$, le raisonnement

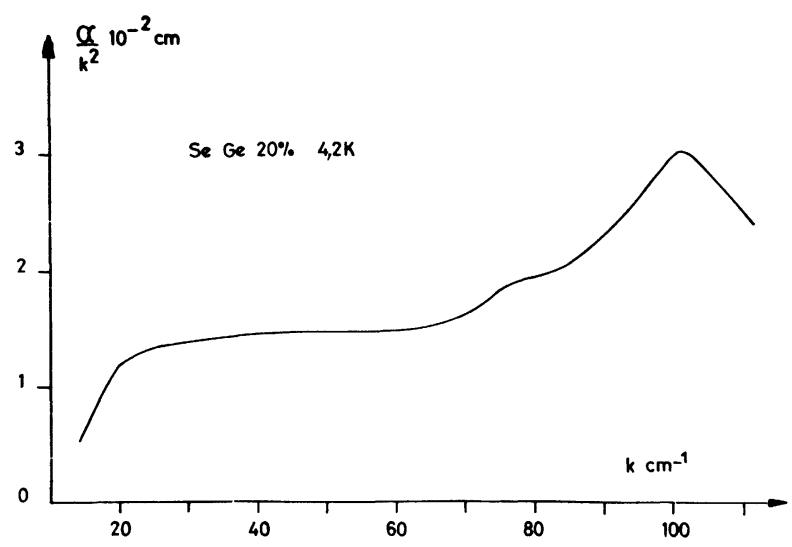

FIG. 6. - Disparition des modes en excès à basse fréquence pour $\mathrm{Se}_{0,8} \mathrm{Ge}_{0,2}$. 
est similaire à celui fait pour la concentration $x=10 \%$. Le pic d'absorption attribué aux chaînes a diminué en intensité et sa position en fréquence est, à $33 \mathrm{~cm}^{-1}$,

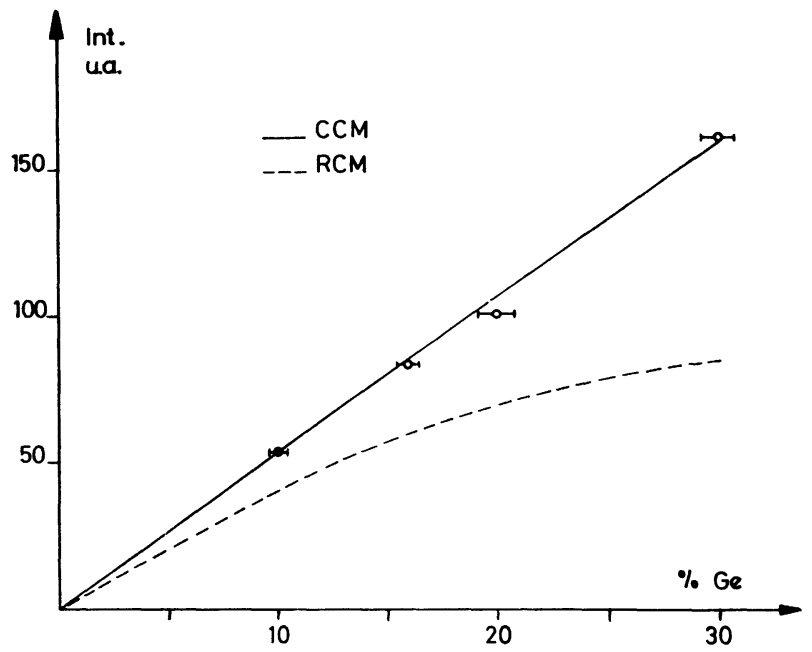

Fig. 7. - Comparaison des modèles R. C. M. et C. C. M. avec les résultats expérimentaux. en accord avec la distance entre seconds voisins qui a augmenté jusqu'à $3,85 \AA$.

Pour la concentration $x=20 \%$, cet excès disparaît (Fig. 6).

L'analyse de tous les spectres met en évidence :

- une décroissance linéaire d'anneaux $\mathrm{Se}_{8}$ jusqu'à une concentration de $8 \%$,

- une décroissance rapide du nombre de chaînes libres pour les concentrations inférieures à $10 \%$. Une analyse plus précise permet de déterminer l'existence de chaînes libres respectivement pour $x=10 \%$ et $x=16 \%$, on trouve ainsi $(4 \%$ et $1 \%$ de sélénium sous forme de chaînes libres),

- une croissance linéaire du nombre de tétraèdre $\mathrm{GeSe}_{4}$ en fonction de la concentration en Ge (Fig. 7).

Ces résultats permettent d'une part de trouver l'évolution du nombre de Se entre site de Ge, d'autre part de choisir entre le modèle R.C.M. (Random crossing model) et le modèle C.C.M. (Chain crossing model) au profit de ce dernier et de préciser la nature du potentiel entre seconds voisins.

\section{Bibliographie}

[1] Bagdade, W., Stolen, R., J. Phys. Chem. Solids 29 (1968) 2001.

[2] Ribeyron, A., Laurant, J. M., Phys. Lett. 49A (1974) 75.

[3] Lucowzky, G., The physics of Se an Fe, Proceedings of the International symposium held at Montréal, Octobre (1967).

[4] Malaurent, J. Non Crystall. Solids, à paraître.

[5] Blanc, J., Brochier, D., Ribeyron, A., Phys. Lett. 31A (1970) 483. 